\title{
TRATAMIENTO ENDOSCÓPICO DEL REFLUJO VESICO URETERAL III-V COMPLICADO EN LACTANTES MENORES DE 1 AÑO
}

\author{
Carlos Miguélez Lago, Jairo Moreno Román, Manuel García Mérida, Eulogio Galiano Duro, \\ Moises Mieles Cerchar y Fernando Ibáñez Cerrato.
}

Urología Pediátrica. Unidad de Cirugía Pediátrica del Hospital Materno Infantil. Complejo Hospitalario Universitario Carlos Haya de Málaga. España.

Clínica Santa Elena. Torremolinos. Málaga. España.

Resumen.- OBJETIVO: Conocer el resultado del tratamiento endoscópico (TE) en lactantes con pielonefritis recurrente y alto grado (G) de Reflujo Vésico Ureteral (RVU).

MÉTODOS. Criterios de inclusión: lactantes de 2-12 meses de edad con RVU G III-V y al menos 2 infecciones de tracto urinario febriles, una durante profilaxis antibiótica (PA). $N=29$ lactantes: 21 del sexo masculino (72.4\%) y 8 del sexo femenino. El RVU fue primario en 19 niños (65.5\%) y secundario en 10. El grado fue III en 13 uréteres (30.9\%), IV en 20 (47.6\%) y $\mathrm{V}$ en 9 (21.4\%). Las sustancias inyectadas fueron por orden cronológico polidimetilxilosano, hidroxiapatita y dextranómero / ácido hialurónico estabilizado no animal. Clasificación de los resultados: Solucionado RVU G O-I, Mejorado G H (control sin PA), Persistente G III-V. En el RVU Persistente de decidió Cirugía Abierta (CA) o repetición de TE en base a los hallazgos cistoscópicos.
Carlos Miguélez Lago

Figurillas, 4.

El Olivar. Torremolinos. 20620. Málaga. (España).

miguelezlago@hotmail.com
RESULTADOS: Hay 1 U G III y 2 U G IV esperando repetición de inyección de TE por lo que no son incluidos, de modo que hay 39 U (92.85\%) válidos para evaluar los resultados finales: $G$ III $12 \mathrm{U}$, con 12 primeras y 4 segundas inyecciones (1.33 inyecciones por uréter): solucionados 10 (83.3\%), Mejoría 7 (8.3\%), CA 7 (8.3\%). GIV 18 U, con 18 primeras y 4 segundas inyecciones (1.22 inyecciones por uréter): Solucionados 14 (77.7\%), Mejoría 4 (22.2\%), No CA. G V 9 $U$, con 9 primeras inyecciones, 4 segundas y 1 tercera 17.55 inyecciones por uréter): Solucionados 5 (55.6\%), Mejoría 1 (17.1\%), CA 3 (33.3\%). CA Evitada: 35 U (89.7\%), G III $91.6 \%$, G N 100\%, G V 66.7\%. Los resultados son mejores en $G$ III que en $V$. La única complicación fue una obstrucción ureteral tratada con éxito con CA.

CONCLUSIONES: El TE puede ser considerado la $7^{a}$ opción terapéutica en lactantes con RVU III-V e ITU a pesar de la PA, porque el TE ha solucionado el RVU en el $74.3 \%$ y evitado al $C A$ en el $89.7 \%$ de los $U$, con un procedimiento minimamente invasivo y escasas complicaciones.

Palabras clave: Lactantes. Reflujo vésicoureteral. Tratamiento endoscópico. Infección de tracto urinario. Pielonefritis. Nefropatía de reflujo. Cicatriz renal.

Summary.- OBJECTIVES: Our aim is to know the results of Endoscopic Treatment (ET) in infants with recurrent pyelonephritis and high grade (G) Vesicoureteral Reflux (VUR).

METHODS: Inclusion criteria: infants 2-12 months old with $G$ III-V VUR and at least 2 pyelonephritis, one of them during antibiotic prophylaxis (AP). N=27 infants: 19 males (70\%) and 8 females. VUR was primary in $17(63 \%)$ and secondary in 10. VUR Grade was III in 12 ureters (U) (32\%), IV 16 (42\%) and V 10 (26\%). Polydimethylsiloxane, Hydroxiapatite and Dextranomer/ Hyaluronic Acid (DAH) were the bulking agents employed. Results Classification: Solved: G O-I.; Improved: $G$ 
II (control without AP); Persistence: III-V. Open Surgery (OS) or repeated ET (1-2) was done depending on cystoscopic findings.

RESULTS: 34 ureters are available for final results; $1 G$ III, 2 GIV and $1 G$ V are waiting for a new injection. G III $11 \mathrm{U}$ : 11 first and 4 second injections (1.36 Injections / ureter): Solved 9 (81.8\%), Improved 1, OS 1 (9\%). GIV 14 U: 14 first 3 second and 1 third injection (1.28 injections / ureter): Solved $10(71.4 \%)$, Improved 4. No OS. G V: 9 U: 9 first, 4 second and 1 third injections (1.55 injections / ureter): Solved 5 (55.6\%), Improved 1, OS 3 (33.3\%). Overall results: Solved: 24 U (70.58\%), Improved: 6 (17.6\%), OS 4 (1 1 . .8\%). OS avoided 30 (88.2\%): G III 91\%, IV 100\% and V $66.7 \%$. Results of $G$ III are better than $G$ V. The only complication was 1 ureteral obstruction treated successfully with open surgery.

CONCLUSIONS: ET can be considered the first therapeutic option in infants with G IIIVV VUR and pyelonephritis in spite of PA, because ET has solved VUR in $70.58 \%$ and avoided $O S$ in $88.2 \%$ with a minimally invasive procedure and low incidence of complications.

Keywords: Infants. Vesicoureteral reflux. Endoscopic Treatment. Urinary tract infection. Pyelonephritis Reflux nephropathy. Renal scar.

\section{INTRODUCCIÓN}

El RVU es el paso retrógrado de orina desde la vejiga hacia los uréteres, desde donde puede llegar a los riñones en la mayoría de los casos (1). Se considera la patología urológica mas frecuente en la infancia (2). En general su incidencia se estima en el $1 \%$, rango $0.4-1.8 \%$ (3). El RVU es más frecuente cuanto menor es la edad, citándose una incidencia general del $1.26 \%$ en el recién nacido (RN) (4). Cuando existe una dilatación del tracto urinario superior (TUS) fetal la incidencia de RVU en el RN es mas alta, $18-25 \%$ (5). También es mas alta la incidencia de RVU en niños con Infección del Tracto Urinario (ITU) 30 $40 \%(5)$.

El RVU es patógeno porque puede producir daño renal, conocido como Nefropatía de Reflujo (NR), que está favorecida por diversos factores: ITU, menor edad (6), mayor grado de RVU (7), y dilatación TUS.

El RVU favorece la repetición de la ITU y viceversa, tendiendo hacia la cronicidad. Se ha observado que mas del $50 \%$ de los niños con RVU III-V diagnosticados por ITU, habían tenido al menos dos ITUS (8).

La incidencia de NR en RVU III-V es elevada, $66 \%$ (7), siendo mayor a mayor grado.

La remisión espontánea (RE) del RVU es mayor cuanto menor es la edad y el grado de RVU. En el primer año de vida se calcula que pueden desaparecer el $70 \%$ de los RVU III (1) y el $40 \%$ de los RVU IV (9).
Debido a la considerable RE del RVU durante el primer año, de tiende a evitar un tratamiento intervencionista durante esta edad. Sin embargo la ITU de repetición en el lactante con RVU de alto grado es una situación que favorece mucho más la aparición y progresión de la NR, por lo que requiere un tratamiento que sea lo mas efectivo y lo menos invasivo posible.

\section{MATERIAL Y MÉTODOS}

Objetivo: Conocer el resultado del tratamiento endoscópico (TE) en lactantes con pielonefritis recurrentes (ITUs febriles) y RVU de alto grado III-V.

Criterios de inclusión: Lactantes de 2-12 meses de edad, con RVU III-V, primario o secundario, con 2 o más ITUs febriles durante la profilaxis antibiótica (PA), con los datos recogidos y un seguimiento mínimo de 4 meses tras TE. El periodo de inclusión es desde el inicio del uso del TE, Marzo 1998, hasta Junio 2007.

Tipo de Estudio: prospectivo de serie de casos que reúnen los criterios de inclusión.

Metodología: Se utilizó una base de datos introducidos por la misma persona en el programa SPSS. Se recogió información referente a: edad, sexo, tipo y grado de RVU, lateralidad, sustancia inyectada, número de inyecciones, resultado y complicaciones.

Valoración de resultados: Reflujo Solucionado RVU 0-I, Mejoría RVU II (controlado sin PA), Persistente RVU III-V.

Aspectos clínicos: El TE se realizó con Cistoscopio compacto $9.5 \mathrm{Fr}$, con aguja metálica, bajo anestesia general, con profilaxis antibiótica, en régimen de Cirugía Mayor Ambulatoria en Hospital de día Pediátrico.

Controles postoperatorios: Visita clínica y ecografía en primero y tercer mes; Cistouretrografía miccional seriada (CUMS) al tercer mes. Se mantuvo la PA monodosis oral diaria hasta el resultado de la CUMS.

En los RVU persistentes se realizó Cirugía Abierta (CA) o repetición del TE dependiendo de los hallazgos cistoscópicos. La repetición del TE fue hasta un máximo de 3 inyecciones.

\section{RESULTADOS}

\section{Casuística}

Entre marzo de 1998 y junio de 2007 se han tratado con TE un total de 377 niños $(\mathrm{N})$ con 598 uréteres (U) con RVU.

De ellos $29 \mathrm{~N}$ fueron menores de 1 año con RVU III-V complicado en $42 \mathrm{U}$ que reunen los criterios de inclusión y son objeto de este estudio. El sexo fue masculino en $21 \mathrm{~N}(72.41 \%)$ y femenino en $8 \mathrm{~N}$. El tipo de RVU fue primario en $19 \mathrm{~N}(65.5 \%)$ y secundario en 10. La distribución 
TABLA I. NÚMERO DE INYECCIONES POR URÉTER SEGÚN GRADOS DE REFLUJO.

\begin{tabular}{|l|c|c|c|c|c|c|}
\hline \multicolumn{2}{|c|}{ № Inyecciones / Grados Refluji } & $\mathbf{1}$ & $\mathbf{2}$ & $\mathbf{3}$ & Total & Inyecciones / Uréter \\
\hline G III $12 \quad 30.76 \%$ & 12 & 4 & & 16 & 1.33 \\
\hline G IV $18 \quad 46.15 \%$ & 18 & 4 & & 22 & 1.22 \\
\hline G V 9 23.07\% & 9 & 4 & 1 & 14 & 1.55 \\
\hline Total Uréteres 39 & 39 & 12 & 1 & 47 & 1.38 \\
\hline
\end{tabular}

de uréteres por grados (G) fue: G III 13 U (30.9\%), G IV 20 U ( $47.6 \%)$, G V 9 U (21.4\%).

Hay $3 \mathrm{U}$ esperando una $3^{\mathrm{a}}$ inyección (G III 1 y $G$ IV 2) por lo que no se incluyen en el resultado final al no haber terminado el tratamiento endoscópico.

Hay $39 \mathrm{U}$ válidos para el análisis de resultados, lo que supone el $92.85 \%$ de los $\mathrm{U}$ iniciales. La distribución por grados fue: G III 12 (30.76\%), G IV 18 (46.15\%) y G V $9(23.07 \%)$.

\section{Número de inyecciones}

En estos 39 uréteres han sido necesarias 52 inyecciones de TE (media de 1.33 inyecciones por uréter) para obtener los resultados que se detallarán a continuación. La distribución de inyecciones según grados se expresa en la Tabla I.

\section{Resultados}

Con 1-3 inyecciones el TE ha conseguido los Resultados que se estratifican por grados en la Tabla II.

En total se ha solucionado el RVU (G 0-I) en $29 \mathrm{U}$ $(74.3 \%)$, se ha mejorado (G II sin PA) en 6 U (15.4\%) y ha persistido el RVU III-V, precisando cirugía abierta (CA) sólo en $4 \mathrm{U}(10.3 \%)$. La CA se ha realizado en $1 \mathrm{U}$ con RVU $G$ III y en $3 \mathrm{U}$ con RVU G V. No ha sido necesaria la CA en ninguno de los $18 \mathrm{U}$ con RVU G IV. Por lo tanto se ha evitado la $\mathrm{CA}$ en el $89.7 \%$ del total: $91.6 \%$ en los $\mathrm{U}$ con RVU G III, $100 \%$ en los G IV y $66.7 \%$ en los G V.

\section{Complicaciones}

Sólo hubo un caso en este grupo de lactantes con $39 \mathrm{U}$ con RVU III-V complicado (2.5\%). Fue un niño de 4 meses con RVU primario $G \mathrm{~V}$, monorreno por agenesia renal contralateral. Se inyectó con $0,8 \mathrm{ml}$ de Deflux $\AA$ en la $1^{\underline{a}}$ y única inyección. En el postoperatorio inmediato presentó anuria persistente durante 48 horas, con elevación de las cifras de creatinina 1,47 MG/DI., y confirmación de la obstrucción con aumento ecográfico de la dilatación Urétero-pielo-calicial. Se trató con CA extirpando el uréter distal y reimplantándolo con técnica de Cohen. La evolución postoperatoria ha sido satisfactoria y sin complicaciones con desaparición del RVU. El seguimiento postoperatorio es de un año.

En este grupo no hubo ningún caso de Reflujo Contralateral ni de Hematuria macroscópica significativa.

\section{DISCUSIÓN}

\section{Disfunción vesical transitoria}

En el recién nacido y lactante del sexo masculino existe un cierto grado de disfunción miccional que es responsable de una mayor incidencia de RVU en este sexo en esta edad. Esta disfunción "parafisiológica" evoluciona

TABLA II. REFLUJO VÉSICO URETERAL IIIV COMPLICADO EN LACTANTESRESULTADOS DEL TRATAMIENTO ENDOSCÓPICO 1-3 INYECCIONES.

\begin{tabular}{|lc|rr|rr|rr|ll|}
\hline \multicolumn{2}{|c|}{ Uréteres } & \multicolumn{2}{|c|}{ Solucionado \% } & \multicolumn{2}{c|}{ Mejoría } & $\%$ & \multicolumn{2}{c|}{ Cirugía abierta $\%$} & \multicolumn{2}{c|}{ Cirugía abierta Evitada \% } \\
\hline Grado III & 12 & 10 & 83.3 & 1 & 8.3 & 1 & 8.3 & 11 & 91.6 \\
\hline Grado IV & 18 & 14 & 77.7 & 4 & 22.2 & 0 & 0 & 18 & 100 \\
\hline Grado V & 9 & 5 & 55.6 & 1 & 11.1 & 3 & 33.3 & 6 & 66.7 \\
\hline Total & 39 & 29 & 74.3 & 6 & 15.4 & 4 & 10.3 & 35 & 89.7 \\
\hline
\end{tabular}


espontáneamente en un proceso uro-neuro madurativo y después del segundo año ya se considera prácticamente como normal. Cuando existe un RVU de alto grado la dinámica vésico esfinteriana se altera mas aún porque hay una "micción intraureteral" y un considerable volumen de orina residual postmiccional. El tratamiento de la disfunción vesical en los niños más jóvenes, con RVU de alto grado no mejora las tasas de resolución espontánea durante una media de tratamiento de 4 años. Estos resultados son contrarios a los vistos en niños mayores con disfunción vesical y reflujo, en quienes el tratamiento de la disfunción acelera la resolución espontánea (10). Los niños con RVU y Disfunción de Vaciado tienen más ITUs importantes (11).

\section{Nefropatía de Reflujo}

La NR en este especial grupo de pacientes está favorecida por la edad menor de un año, el grado de RVU $>$ II, y las ITUs, por lo que se puede denominar RVU Complicado o de Alto Riesgo.

La NR aumenta según grado de RVU: I 10\%, II $17 \%$, III-V $66 \%$ (7). La posibilidad de aparición de Nuevas escaras tras el Diagnóstico del RVU también depende del grado de RVU: I-III 10\%, IV-V 25\% (12).

\section{Resolución espontánea del RVU}

La RE del RVU está condicionada por el grado de RVU, la edad del niño y el tiempo de evolución: es menor con mayor grado, mayor edad y menor tiempo de espera. El RVU desaparece por crecimiento y maduración funcional vesical (1 1). La RE de RVU I-III en niños menores de 5 años, durante 5 años de seguimiento fue en grados I-II $80-82 \%$ y menor en grado III, $46 \%$, pero con aparición de cicatrices en $10-28 \%$ (13). Cuando se incluyen RVU de alto grado y se reduce el tiempo de evolución la RE disminuye considerablemente, con una baja tasa de éxito a un año: $33 \%$ en RVU GII - IV (14). Hay una mayor posibilidad de RE en el primer año de vida (1). En este periodo desaparece el 70\% de los RVU grado III (15) y el $40 \%$ de los IV (9). Durante los primeros años de vida, cada 2 años desaparece un 25 $30 \%$; luego la RE espontánea es rara después de los 10 , y excepcional después de los 15 años (7). Se calcula que un $25 \%$ de RVU llega a la adolescencia (5).

En un estudio de 1500 casos $(16,17,18)$ la RE según grados fue: I $85 \%$ rango (R) $82-92 \%$, || 72\% r 60-81\%, III 56\% r 46-80\%, IV 17\% r 9-25\%, V < 1\%. Hay Diferencia Significativa en la RE anual entre RVU I-III 13,5\% y IV-V 5\% (19) con Nivel de Evidencia $2+$.

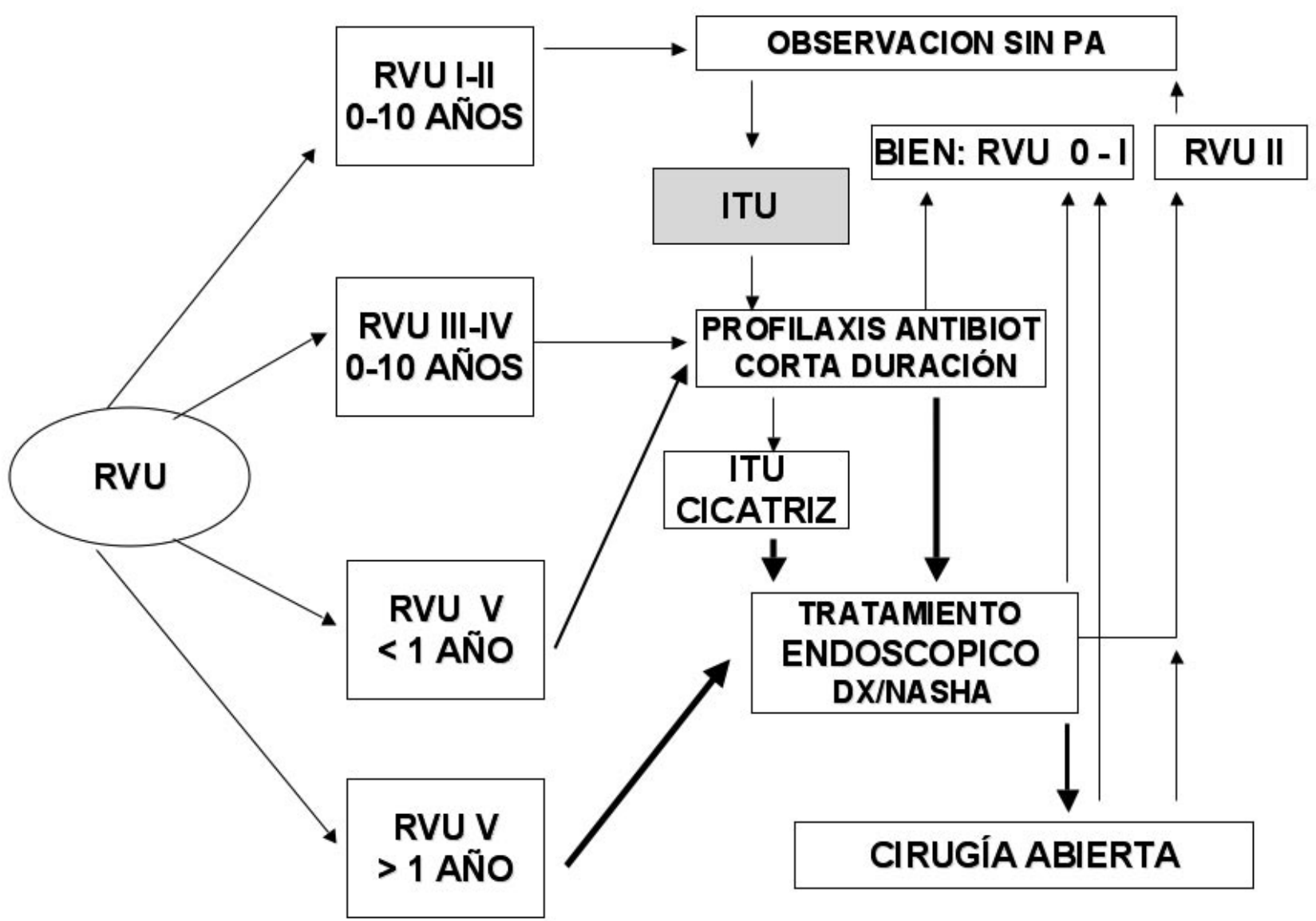

RVU: Reflujo Vésico Ureteral. ITU: Infección del Tracto Urinario. PA: Profilaxis Antibiótica.

DX/NASHA: Dextranómero / Acido hiahulórino estabilizado no animal 
A mayor tiempo mas posibilidad de desaparición, según la edad y el grado (1).

Cuando el RVU desaparece, el tiempo medio $\left(T^{1 / 2}\right)$ es de 2 años, excepto en el primer año que es menos: 1,4 (7). Uniendo tiempo y grado la RE de RVU III-V en 2 años es solo del $20 \%$, y en 5 años del $50 \%(20)$.

La bilateralidad también influye en los grados IIIV: RE unilateral $54 \%$, bilateral $12 \%(7,20)$. menor (11).

Cuando el RVU desaparece el riesgo de ITU es

\section{Observación inicial}

Con toda esta información en nuestra Unidad de Urología Pediátrica de Málaga tenemos el criterio de no tratar el RVU III-V asintomático en el primer año de vida, sólo hacer PA, pero si durante este tiempo aparecen ITUs se considera RVU sintomático complicado y se indica Tratamiento Endoscópico.

\section{Tratamiento Endoscópico}

El TE es un procedimiento sencillo y minimamente invasivo, con hospitalización ambulatoria, con menor costo que la cirugía abierta. La eficacia inicial en RVU III-V es algo menor que con la $C A$, pero es un tratamiento repetible con aumento progresivo de la eficacia (21). La Resolución del RVU con TE decrece con el grado y aumenta con el $\mathrm{n}^{\circ}$ de procedimientos (22). En un metanálisis de Elder, incluyendo todos los grados y edades, la resolución del RVU acumulada, con 1-3 inyecciones, por uréteres fue del $85 \%$ en 8101 unidades renales y por pacientes el éxito fue de $87 \%$ en 5527 niños (23). En el mismo estudio la resolución con la primera inyección fue $78.5 \%$ para grados $1-1 \mathrm{I}, 72 \%$ para el GIII, 63\% GIV y $51 \%$ GV. $(1,22,23)$.

Los resultados en RVU III-V con TE son algo inferiores que con la $\mathrm{CA}$.

El Internacional Reflux Study Comite (IRSC) aporta una resolución del RVU III-V con CA en $91 \%$ para la rama americana y en $81 \%$ par la rama europea (23). Elder hace una revisión bibliográfica de la resolución del RVU con CA resultando: GIII 98.3\%, GIV 98,5\% y GV $80.7 \%$ (23).

La Técnica de Inyección Intrameatal con hidrodistensión (HIT) descrita por Kirsch y colaboradores parece mejorar los resultados del STING cuando los meatos son muy amplios, con hidrodistensión positiva, en hoyo de golf o con divertículo para ureteral (24). El éxito en grado III sube de $78 \%$ a $87 \%$, y sobre todo en grado IV que de $52 \%$ asciende a $71 \%$ (25). La modificación de Kirsch consiste en el implante submucoso intramural, en la pared ureteral, en la parte posterior y si hace falta en la anterior; mejora el resultado respecto a la técnica estándar del 79 al 97\% (26).

En nuestra serie la indicación de CA tras TE ha variado con los años con la aparición de la técnica de HIT y con la publicación de resultados cada vez mejores en RVU IV-V. Hasta 2005 cuando persistía el RVU tras la $1^{a}$ inyección, si el meato era muy patológico (muy amplio, hidrodistendible, con divertículo paraureteral...) y se valoraba como con pocas posibilidades de éxito con $\mathrm{TE}$, en vez de nuevas inyecciones, se indicaba $C A$, por esta razón en el GV hay un 33\% de CA, con solo 4 segundas inyecciones y 1 tercera. A partir del 2005, con la técnica de HIT, se inyectan 1-3 veces hasta que desaparezca el RVU o descienda a Gll. En el total de esta serie se logra una resolución del $74.3 \%$ con RVU 0-I y una mejoría en el $15.4 \%$ con RVU II sin PA; fue necesaria la CA en el $10.3 \%$, por lo que se ha evitado la CA en el $89.7 \%$ de los uréteres. En el futuro los resultados mejorarán por dos motivos principales: menos casos remitidos a CA sin agotar las 3 inyecciones y más experiencia personal y progresión en la curva de aprendizaje.

Puri y cols. (27) tienen unos resultados excelentes. Utilizan como sustancia inyectable el Dextranómero con Ácido Hialurónico estabilizado no animal (DX/NAS$\mathrm{HA})$. Con la $1^{\underline{a}}$ inyección corrigen el RVU en $952 / 1101$ U (86.5\%); el éxito por grados fue II 100\%, III 93.1\%, IV $77.7 \%$ y $\vee 75,9 \%$. De los $149 \mathrm{U}$ restantes el RVU mejoró en el $54 \%$. Con la $2^{\underline{a}}$ inyección corrige el RVU en $130 / 149$ U (87.2\%); el éxito por grados fue III 90\%, IV $85.3 \%$ y V $100 \%$. Con la $3^{\text {a }}$ inyección corrige el RVU en $19 / 19$ U (100\%); el éxito por grados fue III 100\% y IV $100 \%$. Con $1-3$ inyecciones han conseguido un $100 \%$ de éxito, de los que sólo $42 \mathrm{U}$ quedaron con grado I (3.8\%), en los $1059 \mathrm{U}$ restantes el RVU desapareció por completo (27).

En la serie de Yu y Roth (22) con 162 uréteres, utilizando las técnicas de STING y de HIT según los hallazgos endoscópicos, tras la $1^{\underline{a}}$ inyección desapareció por completo el RVU en el $82,2 \%$ de los pacientes y en el $86,9 \%$ de los uréteres. Tras la $2^{\mathrm{a}}$ inyección la desaparición del RVU en el total de la serie asciende al $90,7 \%$ de los pacientes y al $92,6 \%$ de los uréteres.

\section{Factores influyentes en el resultado del TE}

Puri y cols mediante un análisis de regresión logística, estudian las variables responsables del fallo inicial del $T E$, identificando en primer lugar el mayor grado de RVU inicial y después la menor edad de los pacientes (27).

Subramanian y cols en un estudio de 60 casos de RVU > II o RVU sintomático, con 27 niños y 33 niñas tratados con (DX/NASHA) observan que la resolución del RVU a la $1^{\underline{a}}$ inyección es mayor en el sexo femenino, $81 \%$, que en el masculino, $53 \%$. Con una $2^{a}$ inyección la resolución sube a $92 \%$ en niñas, pero no sube en niños (28).

La experiencia es también un factor de gran importancia en el éxito el TE. Kirsch analiza este dato en sus 180 primeros casos: el éxito a la $1^{\underline{a}}$ Inyección en los primeros 20 pacientes fue $60 \%$, mientras que en los 20 últimos fue $80 \%(26)$. Lorenzo y cols demuestran estadísticamente que es uno de los factores más importantes de éxito (29).

En nuestra serie los resultados incluyen la totalidad de los casos desde el inicio de la curva de aprendizaje. El TE fue realizado por 4 miembros del equipo de Urología Pediárica, por lo que la experiencia acumulada por Urólogo es relativamente menor. En nuestra serie se unen todos los 
factores negativos predisponentes, ya que solo incluye niños menores de un año, con RVU III-V, el $70 \%$ son del sexo masculino y tratados desde el inicio del aprendizaje del TE.

\section{Complicaciones del TE}

Hay menos numero y menos graves complicaciones con TE que con CA $(23,30)$. Elder $(26)$ refiere en su metanálisis 42 Hidronefrosis tras TE en $5295 \mathrm{U}, 0.8 \%$, de los que precisaron intervención 24 , es decir el 0,45\%. La incidencia de obstrucción varía según autores entre el $1.7 \%$ y el $0.006 \%$ (26). Puri en una revisión multicéntrica encuentra estenosis de meato en menos del 0,5\% (31). Vandersteen y colaboradores (32) en una reciente publicación refieren una incidencia de $\mathrm{OU}$ del $0.7 \%$

En nuestra experiencia sólo ha habido $2 \mathrm{U}$ con obstrucción en el total de 598 U con TE (0.33\%), incidencia que está por debajo de la media. Uno de los casos de obstrucción ocurrió en este grupo seleccionado de lactantes con $39 \mathrm{U}$ con RVU III-V complicado (2.5\%), por lo que parecería que la obstrucción pudiera ser mayor en este grupo especial de pacientes, pero al ser una serie relativamente corta no se pueden sacar conclusiones significativas. No ha aparecido ningún RVU contralateral nuevo tras TE en este grupo de pacientes. No se intentó la colocación temporal endoscópica de un catéter doble $\mathrm{J}$, por ser monoreno y obligar a otro procedimiento con anestesia general para la extracción, sin la certeza de solucionar la obstrucción.

Las complicaciones del TE son menores que las descritas con la CA. Elder (23) refiere tras CA obstrucción ureteral 2-9\%, nuevo RVU contralateral 9.1\%, persistencia de RVU 3-4\% (23).

\section{Ventajas del TE}

Alta incidencia de curación inmediata que no depende del cumplimento terapéutico a largo plazo y con muy escasos efectos adversos, se puede repetir con facilidad en caso de fallo tras la primera inyección (27). Para Stenberg y läckgren además de una mayor reducción de las ITUs febriles, el TE es un procedimiento minimamente invasivo, ambulatorio, mejor costo/beneficio, y no deja cicatriz externa (8).

Relacionándolo con la PA a largo plazo, disminuye la aparición de resistencias bacterianas, evita los problemas de la difícil adhesión a la administración prolongada de antibióticos, tiene una mayor desaparición del RVU en menor tiempo y evita las CUMS de repetición, que es la vivencia peor valorada por niños y padres (8).

Capozza añade que el TE tiene la ventaja de un excelente postoperatorio con vida normal en 24 horas (25).

\section{Elección de tratamiento}

Los padres prefieren el TE antes que la PA y que la CA, como se demuestra en una encuesta de Capozza: el $80 \%$ prefirió el TE, $5 \%$ la PA, $2 \%$ la CA y $13 \%$ no manifestaron preferencia (14).

Para la elección del tratamiento Yu y Roth también se basan en la opinión de los padres exponiéndoles las caracte- rísticas de las 3 opciones, TE, CA, PA, y refieren que el TE con Deflux $\circledast$ ha sido la 1ª opción elegida por los padres (22).

La elección del tratamiento se debe hacer atendiendo a las características de cada paciente y de su familia, explicando a los padres la efectividad del TE como alternativa a PA a largo plazo (26) y escuchando sus preferencias.

Es importante informar también de las posibilidades de desaparición espontánea del RVU sobre todo en el primer año de la vida, así como del riesgo de ITUs y nefropatía de reflujo.

\section{Algoritmo terapéutico}

Dada la mayor tasa de desaparición del RVU en este periodo, si el lactante no presenta ITUs ni retraso ponderal, en general preferimos esperar con PA, ver evolución y controlar con CUMS, Ecografía, DMSA Tc 99 y analítica un año después del CUMS que diagnosticó el RVU. Si persiste el RVU III-V aconsejamos TE.

Nuestro esquema de toma de decisiones se expresa en el algoritmo terapéutico de la Tabla III, que es una modificación del descrito por Läckgren y cols en 2003 (21). La principal diferencia es hacer siempre TE antes que la CA en los RVU V mayores de 1 año, y no hacer PA de entrada en los RVU I-II.

Una vez indicado el TE, preferimos utilizar el DX/ NASHA (Deflux®) ya que está aprobado por la FDA, no migra, no tiene propiedades inmunológicas, es biodegradable, no se han descrito efectos adversos (22).

\section{CONCLUSIONES}

El TE del RVU III-V complicado durante el primer año de vida ha logrado evitar la CA en $89.7 \%$ con una media de inyecciones por uréter de 1.33.

El TE del RVU III-V complicado con ITUs durante el primer año de vida es la primera opción terapéutica frente a la PA y la CA, pues permite buenos resultados con escasas complicaciones, y relativamente bajo número de procedimientos.

La CA del RVU III-V complicado en el primer año de vida se indica tras fracaso del TE.

\section{BIBLIOGRAFIA y LECTURAS RECOMENDADAS ( 'lectura de interés $\mathrm{y}^{* *}$ lectura fundamental)}

1. MIGUÉLEZ, C.; GARCÍA MÉRIDA, M.; RECOBER, A.: "Reflujo Vésico Ureteral". Libro de Residentes de la Asociación Española de Urología. Coordinador Castiñeiras Fernandez J. Grupo ENE. Pp 281-302 Madrid. 2007

**2. ELDER, J.S.; PETERS, C.A.; ARANT, B.S. Jr. y cols.: "Pediatric Vesicoureteral Reflux Guidelines Panel summary report on the management of primary vesicoureteral reflux in children”. J. Urol., 157: 1846, 1997. 
3. BELMAN, A.B.: "Vesicoureteral reflux". Pediat. Clin. North Am., 44: 1171, 1997.

4. TSAI, J.D.; HUANG, F.Y.; TSAI, J.C.: “Asymptomatic vesicoureteral reflux detected by neonatal ultrasonographic screening”. Pediatr. Nephrol., 12: 206, 1998.

5. GODLEY, M.L.: "Vesicoureteral Reflux: Pathophysiology and experimental studies". Pediatric Urology. Gearhart JP, Rink RC, Mouriquand PDE, Eds. WB Saunders, pp 359-381, Philadelphia. 2001.

**6. RANSLEY, P.G.; RIDSON, R.A.; GODLEY, M.L.: "High pressure sterile vesicoureteral reflux and renal scarring: An experimental study in the pig and minipig”. Contrib. Nephrol., 39: 320, 1984.

7. GREENFIELD, S.P.; LEWIS, W. 3rd.; PERRY, B. y cols.: "Regional renal blood flow measurements using radioactive microspheres in a chronic porcine model with unilateral vesicoureteral reflux". J. Urol., 154: $816,1995$.

*8. STENBERG, A.; LÄCKGREN, G.: “Treatment of Vesicoureteral Reflux. Children using stabilized nonanimal hyaluronic acid/dextranomer gel (Nashda/ Dex): A long term observational study". J. P. Urol., 3: 80, 2007.

9. SCOTT, J.S.: "Fetal ureteric reflux: A follow up study". Br. J. Urol., 71: 481, 1993.

10. SILLÉN, U.; HOLMDAHL, G.; HELLSTRÖM, A.L. y cols.: "Treatment of bladder dysfunction and high grade vesicoureteral reflux does not influence the spontaneous resolution rate". J. Urol., 177: 325, 2007.

**11. ELDER, J.S.: "Nonsurgical management of vesicoureteral reflux". Clinical Pediatric Urology Docimo SG, Canning DA, Khoury AE, Eds. Informa Healthcare UK Ltd, pp 663-672, London, 2007.

12. GOLDRAICH, N.P.; GOLDRAICH, I.H.: "Follow up of conservatively treated children with high and low grade vesicoureteral reflux: A prospective study". J. Urol., 148: 1688, 1992.

13. ARANT, B.S.J.: "Medical management of mild and moderate vesicoureteral reflux: Follow up studies of infants and young children. A preliminary report of the southwest pediatric nephrology study group". J. Urol., 148: 1683, 1992.

*14. CAPOZZA, N.; LAIS, A.; MATARAZZO, E. y cols.: "Treatment of vesico-ureteric reflux: A new algorithm based on parental preference". BJU., 92: 285, 2003.

*15. TAMMINEM-MOBIUS, T.; BRUNIER, E.; EBEL, K.D. y cols.: "Cessation of vesicoureteral reflux for 5 years in infants and children allocated to medical treatment. The international reflux study in children (European Branch)". J. Urol., 148: 1662, 1992.

16. GODLEY, M.L.; RANSLEY, P.G.; PARKHOUSE, H.F. y cols.: "Quantitation of vesico-ureteral reflux by radionucleide cystographie and urodynamics". Pediatr. Nephrol., 4: 485, 1990.

*17. WEISS, R.; DUCKETT, J.; SPITZER, A.: "Results of a randomized clinical trial of medical versus surgical management of infants and children with grades III and IV primary vesicoureteral reflux (United States). The international reflux study in children". J. Urol., 148: 1667, 1992.
18. OLBING, H.; CLAESSON, I.; EBEL, K.D. y cols.: "Renal scars and parenchymal thinning in children with vesicoureteral reflux: A 5-year report of the international reflux study in children (European Branch)". J. Urol., 148: 1653, 1992.

19. SCHWAB, C.V.; WU, H.S.; SELMAN, H. y cols.: "Spontaneous resolution of vesicoureteral reflux: A 15year perspective". J. Urol., 168: 2594, 2002.

20. McLOIRE, G.A.; McKENNA, P.H.; JUMPER, B.M. y cols.: "High grade vesicoureteric reflux: Analysis of observational therapy”. J. Urol., 144: 537, 1990.

*21. LÄCKGREN, G.; LOTTMANN, H.; HENSLE, T. y cols.: "Endoscopic treatment of vesico ureteral reflux and urinary incontinence in children". AUA Series., 37: 294, 2003.

22. YU, R.N.; ROTH, D.R.: "Treatment of vesico ureteral reflux using endoscopic injection of non animal stabilized hyaluronic acid/dextranomer gel: Initial experience in pediatric patients by a single surgeon". Pediatrics., 118: 698, 2006.

*23. KIRSCH, A.J.; PEREZ-BRAYFIELD, M.R.; SMITH, E.A. y cols.: "The modified STING procedure to correct vesicoureteral reflux: Improved results with submucosal implantation within the intramural ureter". J. Urol., 171: 2413, 2004.

**24. ELDER, J.S.; MIREYA DIAZ.; CALDAMONE, A.A. y cols.: "Endoscopic therapy for vesicoureteral reflux: A meta-analysis. I. Reflux resolution and urinary tract infection". J. Urol., 175: 716, 2006.

25. CAPOZZA, N.; LAIS, A.; NAPPO, S. y cols.: "The role of endoscopic treatment of vesicoureteral reflux: A 17-year experience". J. Urol., 172: 1626, 2004.

26. CALDAMONE, A.A.: "Injection therapy for vesicoureteral reflux". Clinical Pediatric Urology Docimo SG, Canning DA, Khoury AE, Eds. Informa Healthcare UK Ltd, pp 691-712, London, 2007.

27. PURI, P.; PIKER, M.; MOHANAN, N. y cols.: "Subureteral dextranomer/hyaluronic acid injection as first line treatment in the management of high grade vesicoureteral reflux". J. Urol., 176: 1856, 2006.

28. SUBRAMANIAM, R.; TURNER, A.; KOUSIDIS, G. y cols.: "Influence of the gender on the outcome of endoscopic correction in vesicoureteric reflux". J. P. Urol., 3: 516, 2007.

29. LORENZO, A.J.; PIPPI SALLE, J.; BARROSO, U. y cols.: "What are the most powerful determinants of endoscopic vesicoureteral reflux correction? Multivariate analysis of single institution experience during 6 years". J. Urol., 176: 1851, 2006.

*30. DE LA PEÑA, E.: "Tratamiento del reflujo vesicoureteral primario en la infancia: Comparación de dos revisiones sistemáticas”. Actas Urol. Esp., 29: 138, 2005.

31. PURI, P.: "Endoscopic treatment of vesicoureteral reflux". Pediatric Urology, Gearhart JP, Rink RC, Mouriquand PDE, Editors. WB Saunders, pp 411-420, Philadelphia, 2001.

32. VANDERSTEEN, D.R.; ROUTH, J.C.; KIRSCH, A.J. y cols.: "Postoperative ureteral obstruction after subureteral injection of dextranomer/hyaluronic acid copolymer”. J. Urol., 176: 1593, 2006 